\title{
Effect of Conformational Heterogeneity on Excitation Energy Transfer Efficiency in Directly meso-meso Linked Zn(II) Porphyrin \\ Arrays
}

Tae Kyu Ahn, ${ }^{1,2}$ Zin Seok Yoon, ${ }^{1}$ In-Wook Hwang, ${ }^{1}$ Jong Kuk Lim, ${ }^{1,2}$ Hanju Rhee, ${ }^{3}$

Taiha Joo, ${ }^{3}$ Eunji Sim, ${ }^{1}$ Seong Keun Kim, ${ }^{2}$ Naoki Aratani,${ }^{4}$ Atsuhiro Osuka, ${ }^{4, *}$ and Dongho Kim ${ }^{1, *}$

${ }^{I}$ Department of Chemistry and Center for Ultrafast Optical Characteristic Control,

Yonsei University, Seoul 120-749, Korea ${ }^{2}$ School of Chemistry, Seoul National

University, Seoul 151-747, Korea ${ }^{3}$ Department of Chemistry, Pohang University of

Science and Technology, Pohang 790-784 ${ }^{4}$ Department of Chemistry, Kyoto University,

Sakyo-ku, Kyoto 606-8502, Japan

E-mails: osuka@kuchem.kyoto-u.ac.jp, dongho@yonsei.ac.kr 
Synthesis. Details of synthesis was explained elsewhere. ${ }^{2} p-(4,4,5,5-$ Tetramethyl-1,3,2-dioxaborolan-2-yl)-benzaldehyde was prepared in 92\% yield by pinacol protection of 4 -formylphenylboronic acid. The porphyrin boronate $\mathbf{1}$ was prepared in $13 \%$ yield from the acid catalyzed condensation of 3,5dioctyloxybenzaldehyde and the protected boronate with 2.0 equiv. 2,2'dipyrromethane followed by oxidation with 3 equiv DDQ. Subsequent bromination of 1 with 2.2 equiv. NBS followed by zinc metallation yielded $\mathrm{Zn}(\mathrm{II})$ dibromoporphyrin 2 in $92 \%$ yield. Sonogashira coupling of the porphyrin 2 with an excess of phenylacetylene (bis(triphenylphosphine) palladium(II) chloride and CuI, triethylamine/toluene, at $50{ }^{\circ} \mathrm{C}$, for $3 \mathrm{~h}$ ) gave 5,15-bisphenylethynylated boronate $\mathrm{Zn}(\mathrm{II})$ porphyrin 3 in $64 \%$ yield. Finally, Suzuki cross-coupling reaction of the porphyrin 3 with Z1B, Z2B, Z3B, Z6B, Z12B, and Z24B (10 mol\% $\mathrm{Pd}\left(\mathrm{PPh}_{3}\right)_{4}, 3$ equiv $\mathrm{Cs}_{2} \mathrm{CO}_{3}, \mathrm{DMF} /$ toluene, at $80{ }^{\circ} \mathrm{C}$, for $4 \mathrm{~h}$ ) gave models Z1A, Z2A, Z3A, Z6A, Z12A, and Z24A in 10 77\% yields. Models ZnA were difficult to separate over the usual silica gel column but were obtained in a pure form by using the recycling preparative GPCHPLC. In the case of Z24A, as many as 16 recycling over our GPC-HPLC setup enabled the isolation of pure Z24A in 28\% (Supporting Information 1). 5,15-Bisphenylethynyl10,20-bis(3,5-dioctyloxyphenyl) $\mathrm{Zn}(\mathrm{II})$ porphyrin 4 was prepared as a reference molecule. All new compounds were fully characterized by ${ }^{1} \mathrm{H}$ NMR spectra, FAB or MALDI-TOF mass spectroscopy, UV-vis absorption spectroscopy, and GPC analysis. Among these, the long porphyrin arrays Z12A and Z24A contain 13 and 25 porphyrin units in a linear fashion with a molecular length of 113 and $217 \AA$, respectively. The corresponding parent ions were clearly detected at $m / e=13423$ (calcd for $\mathrm{C}_{832} \mathrm{H}_{1044} \mathrm{~N}_{52} \mathrm{O}_{50} \mathrm{Zn}_{13}, 13423.9$ ) and $m / e=25862$ (calcd for $\mathrm{C}_{1600} \mathrm{H}_{2028} \mathrm{~N}_{100} \mathrm{O}_{98} \mathrm{Zn}_{25}, 25865.1$ ) in the MALDI-TOF mass spectra. 


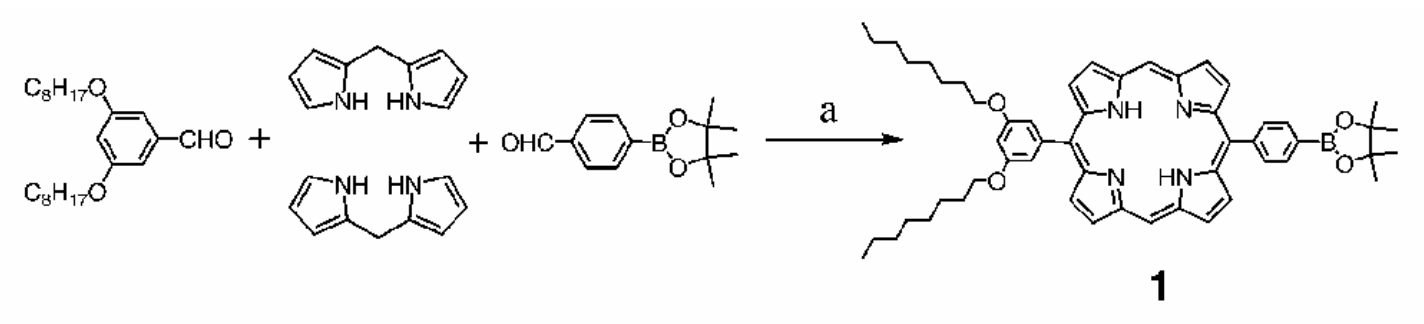

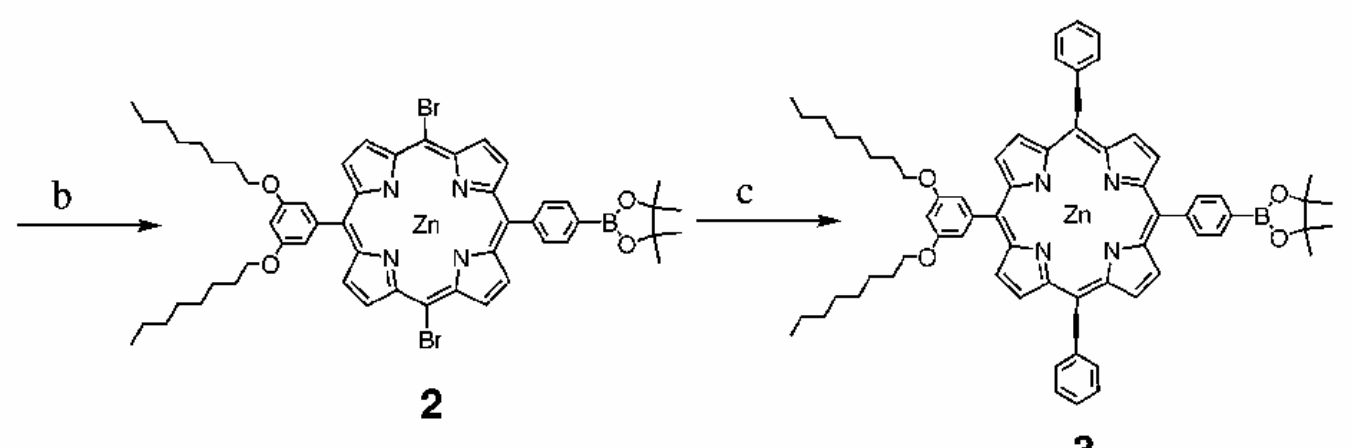

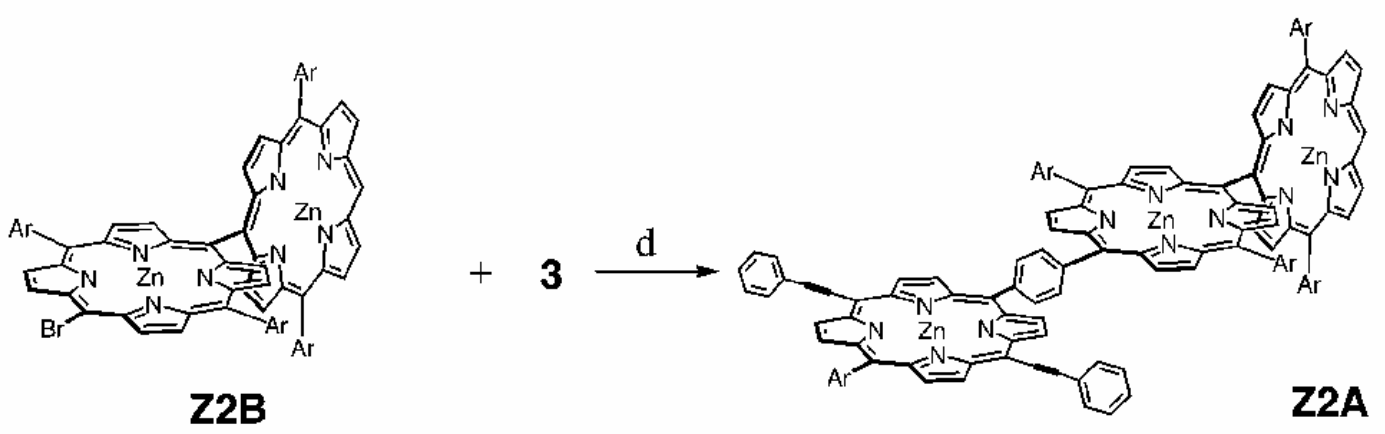

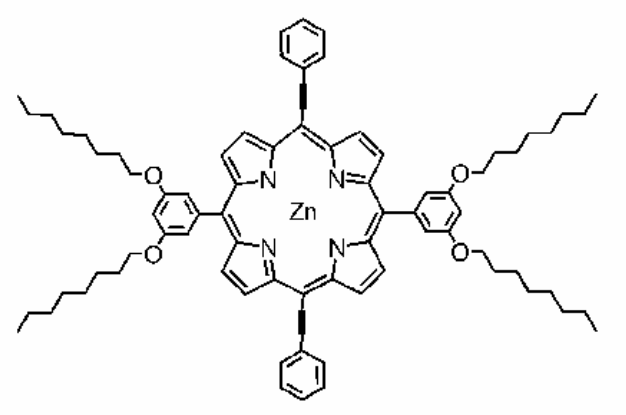




\section{Coherence Length $(L)$ of Orthogonal Porphyrin Arrays (Zn)}

The systematic changes of the absorption bands can be explained by the simple point dipole exciton model. The splitting energy $(\Delta \mathbf{E})$ for longer arrays can be given as follows

$$
\Delta E=2 V \cos \left(\frac{\pi}{N+1}\right)
$$

where $N$ represents the number of porphyrin units and $V$ is the coupling strength. The observed Soret band splitting exhibits a good linear correlation as predicted by eq 1 . The splitting energy $\Delta E$ can be determined by the observed energy difference between the low- and high-energy Soret bands. When $\Delta E$ data were plotted to eq. 1 , we obtained a straight line with a slope of $2 V=4300 \mathrm{~cm}^{-1}$. In a similar analogy, the energy differences between the $\mathrm{Q}(1,0)$ bands of $\mathbf{Z 1}$ and longer porphyrin arrays ( $\mathbf{Z 2}-\mathbf{Z 1 6})$ give rise to the best-fitted lines with a slope of $2 \mathrm{~V}=1140 \mathrm{~cm}^{-1}$. According to the empirical formula of exciton coherence for one-dimensional linear array developed by Kakitani et al., ${ }^{1}$ the coherence length $(L)$ can be estimated as follows

$$
\boldsymbol{L}=1.38+1.33 \frac{\boldsymbol{V}}{\gamma}
$$

where $\gamma$ is the homogeneous broadening.

By using the spectral bandwidth of the Q-band of $\mathrm{Zn}$ (II)TPP at $103 \mathrm{~K}, \gamma$ can also be estimated to be $250 \mathrm{~cm}^{-1}$. Consequently, we obtained $L=4.5$ porphyrin units in $\mathrm{Zn}$. 


\section{Spectral Overlap and Radiative Rate Constants}

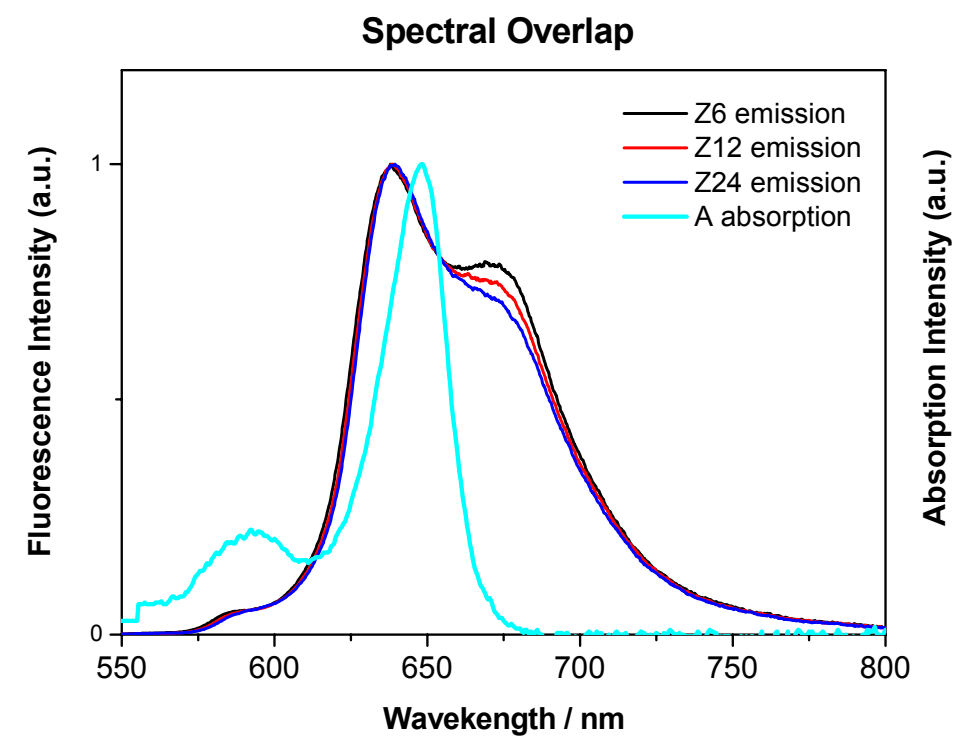

Figure S1. Normalized fluorescence and absorption spectra of donors (Z6, Z12, Z24) and acceptor (A), respectively, in toluene to compare the spectral overlap. Little differences are observed in the spectral overlap with the change of donor.

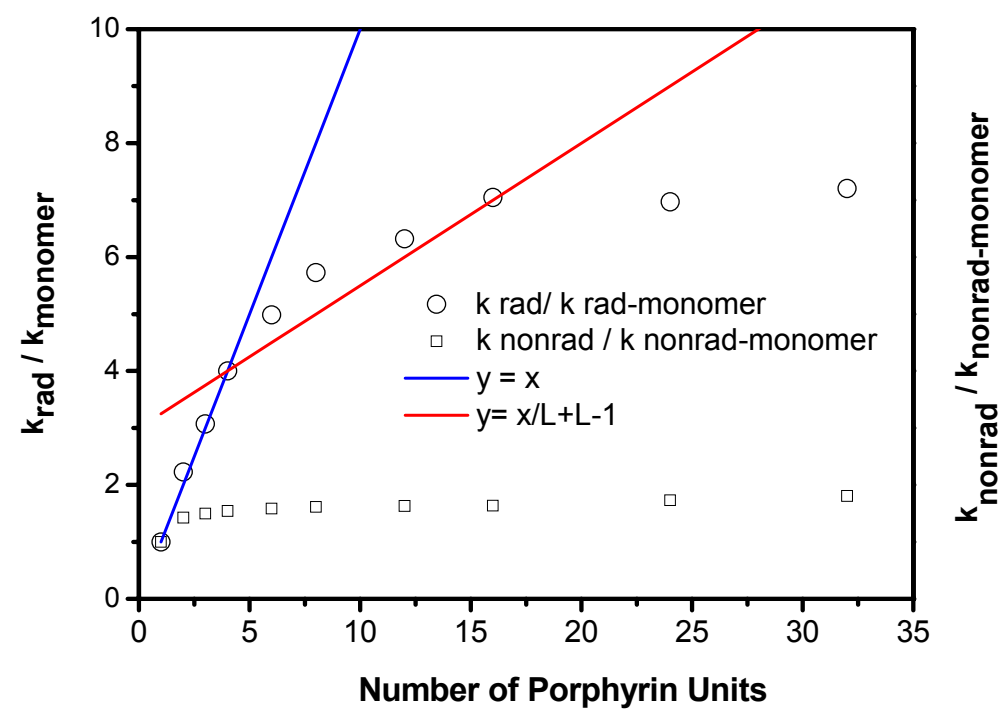

Figure S2. Estimated radiative $(\bigcirc)$ and non-radiative $(\square)$ rate constants of porphyrin arrays. The blue line indicates that the coherence length $(L)$ equals to four porphyrin units, and the red line from the hypothesis of coherent domain emission matches well 
from $\mathbf{Z 4}$ to Z16. Although the non-radiative rate constants show little changes, the radiative rate constants reach maximum around $\mathbf{Z 1 6}$ and saturate over Z24. Longer arrays than $\mathbf{Z 2 4}$ show large discrepancies between the correlated straight line and the observed radiative rate constants, indicating large conformational heterogeneity. 


\section{Excitation Energy Transfer Rates.}

The rate constant for the EET obtained is given by

$$
k_{N}=\frac{4 \pi J^{2}}{\hbar(N+1)}\left(\frac{R_{1}}{R}\right)^{6} \cot ^{2}\left(\frac{\pi}{2 N+2}\right) \int I_{a}(E) L_{N}(E) d E
$$

where $I_{a}(E)$ and $L_{N}(E)$ represent respectively the absorption spectrum of the acceptor porphyrin and the fluorescence spectrum of the array of $N$ porphyrins as the donor, both being normalized as

$\int \operatorname{Ia}(E) d E=\int L N(E) d E=1$

For $N=1$, eq 3 reduces to

$k_{1}=\frac{2 \pi}{\hbar} J^{2} \int \operatorname{Ia}(E) L 1(E) d E$.

From the Förster energy transfer model, eq S3 can be rearranged as,

$$
\frac{k_{N}}{k 1}=\frac{2}{N+1}\left(\frac{R 1}{R}\right)^{6} \cot ^{2}\left(\frac{\pi}{2 N+2}\right) \frac{\int \operatorname{Ia}(E) L N(E) d E}{\int \operatorname{Ia}(E) L 1(E) d E} .
$$

From the hopping model, excitation energy transfer rate can be expressed as, $k_{N}=k_{1} / N$.

Considering the coherence length (L), eq S3 can be divided doubly as,

$$
\begin{aligned}
& \frac{k_{N}}{k_{1}}=\frac{2}{N+1}\left[\sum_{n=1}^{N} \sin \left(\frac{\pi}{N+1} n\right)\left(\frac{R_{1}}{R_{n}}\right)^{3}\right]^{2} \frac{\int I_{a}(E) L_{N}(E) d E}{\int I_{a}(E) L_{1}(E) d E}, \text { for } N<L, \\
& k_{N} / k_{1}=\left(k_{L} / k_{1}\right) /(N-L+1), \quad \text { for } N>L .
\end{aligned}
$$

As we reported previously, eqs 8 and 9 give a good accordance between the calculated and experimental energy transfer rates (Table S1). 
Table S1. Observed and Calculated EET Rate Constants.

\begin{tabular}{cccccc} 
Model & $R(\AA)^{\mathrm{a}}$ & $k_{o b s}{ }^{-1}(\mathrm{ps})^{\mathrm{b}}$ & $k_{N}^{-1}(\mathrm{ps})^{\mathrm{c}}$ & $k_{N}^{-1}(\mathrm{ps})^{\mathrm{d}}$ & $k_{N}{ }^{-1}(\mathrm{ps})^{\mathrm{e}}$ \\
Z1A & 12.7 & $2.5 \pm 0.1$ & - & - & - \\
Z2A & 16.9 & $3.3 \pm 0.2$ & 6.9 & 5.0 & 3.4 \\
Z3A & 21.0 & $5.5 \pm 0.5$ & 20 & 7.5 & 5.9 \\
Z6A & 33.6 & $21 \pm 2$ & 160 & 15 & 23 \\
Z12A & 58.6 & $63 \pm 5$ & 2300 & 30 & 70 \\
Z24A & 109 & $108 \pm 7$ & 49000 & 60 & 163 \\
\hline
\end{tabular}

${ }^{a}$ Center to center distances between donor and acceptor.

${ }^{b}$ EET rate constants measured by the transient absorption spectra.

${ }^{c}$ EET rate constants calculated by eq. S6.

${ }^{d}$ EET rate constants calculated by eq. S7.

${ }^{e}$ EET rate constants calculated by eqs. S 8 and $\mathrm{S} 9$ for $L=4$. 
Table S2. Fluorescence Lifetimes of Z256 and Z512

\begin{tabular}{lllll}
\hline Sample & Wavelength $(\mathrm{nm})$ & $\tau_{1}(\mathrm{~ns}, \%)$ & $\tau_{2}(\mathrm{~ns}, \%)$ & $\tau_{\text {ave }}(\mathrm{ns})$ \\
\hline \multirow{2}{*}{$\mathbf{Z 2 5 6}$} & 600 & $1.04(25)$ & $0.138(75)$ & 0.36 \\
& 650 & $1.06(27)$ & $0.144(73)$ & 0.39 \\
& 705 & $1.07(31)$ & $0.148(69)$ & 0.43 \\
\hline \multirow{2}{*}{$\mathbf{Z 5 1 2}$} & 600 & $0.965(16)$ & $0.124(84)$ & 0.26 \\
& 650 & $0.902(14)$ & $0.114(86)$ & 0.22 \\
& 705 & $0.940(20)$ & $0.114(80)$ & 0.28 \\
\hline
\end{tabular}




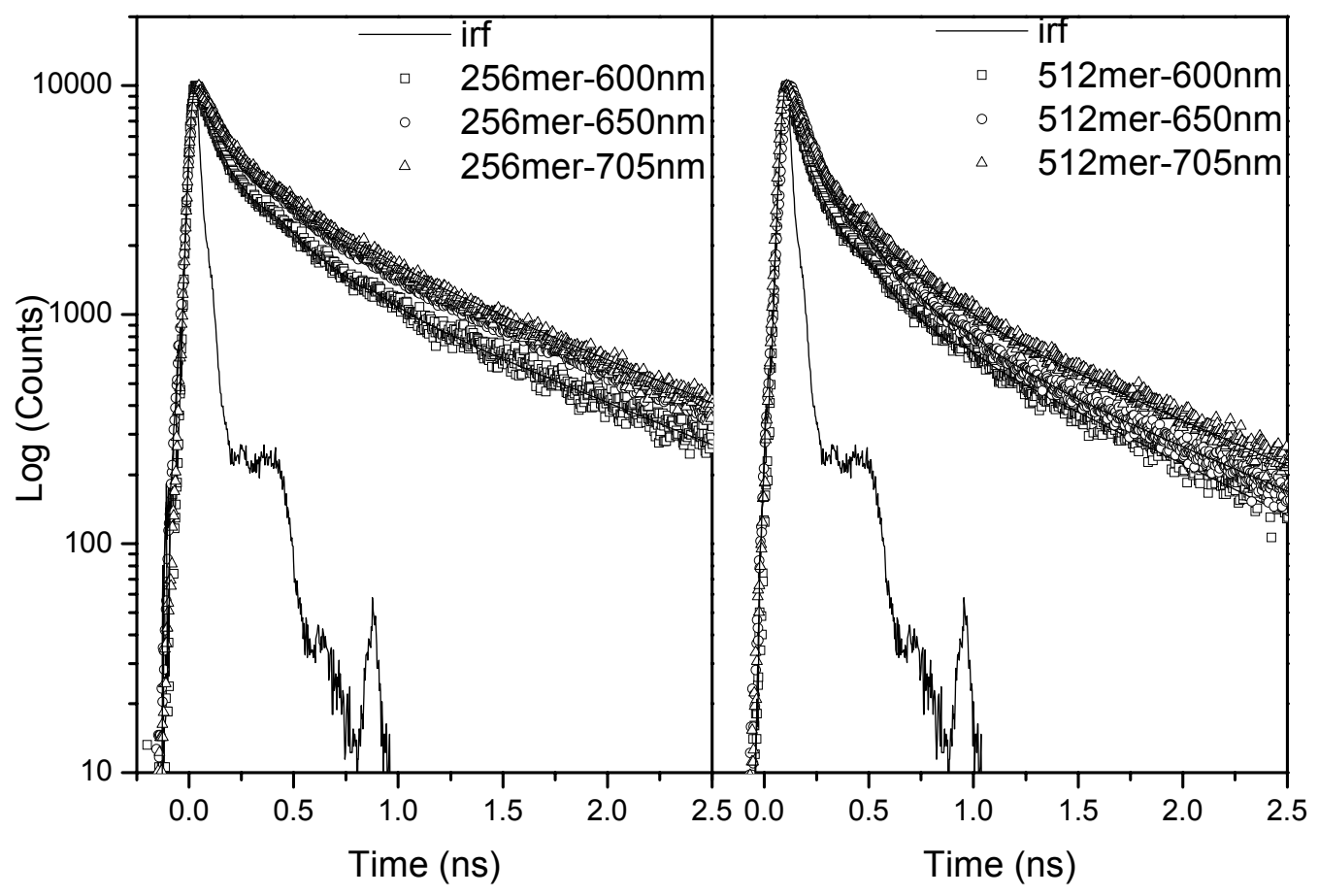

Figure S3. Fluorescence decay profiles of Z256 (left) and Z512 (right) measured at 600, 650 , and $700 \mathrm{~nm}$. 


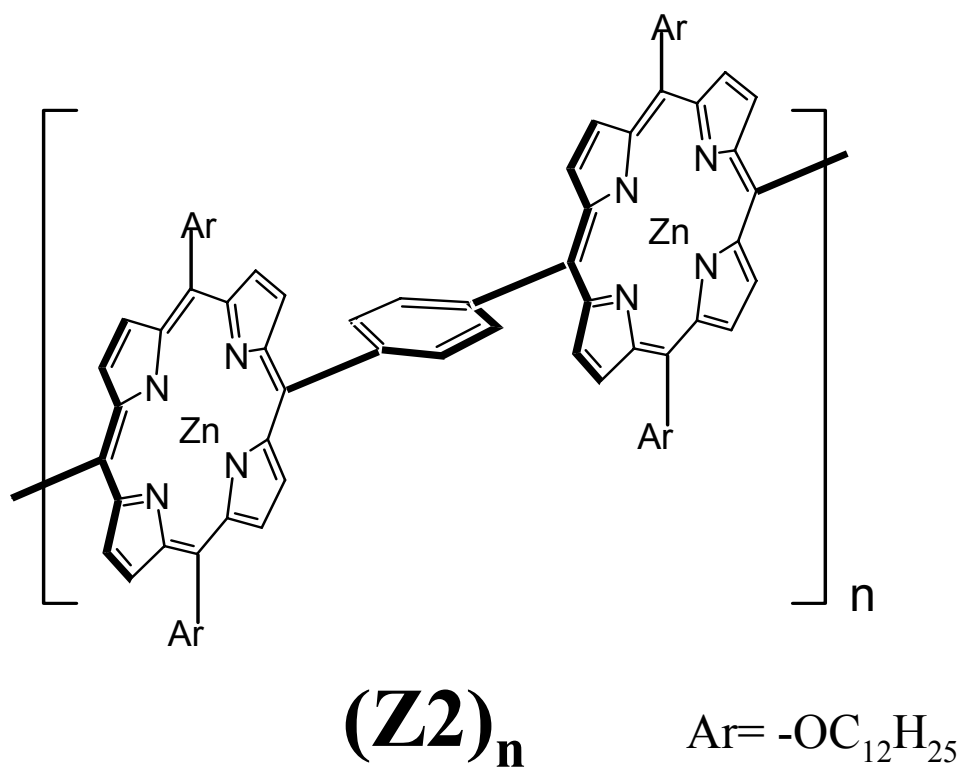

$\begin{array}{llll}(\mathbf{Z 2})_{1} & \mathrm{n}=1 & (\mathbf{Z 2})_{\mathbf{6}} & \mathrm{n}=6 \\ \mathbf{( Z 2})_{\mathbf{2}} & \mathrm{n}=2 & (\mathbf{Z 2})_{\mathbf{8}} & \mathrm{n}=8 \\ \mathbf{( Z 2 ) _ { 3 }} & \mathrm{n}=3 & (\mathbf{Z 2})_{\mathbf{1 2}} & \mathrm{n}=12 \\ \mathbf{( Z 2 )}_{\mathbf{4}} & \mathrm{n}=4 & (\mathbf{Z 2})_{\mathbf{1 6}} & \mathrm{n}=16\end{array}$

Chart S1. Molecular structures of (Z2) . 


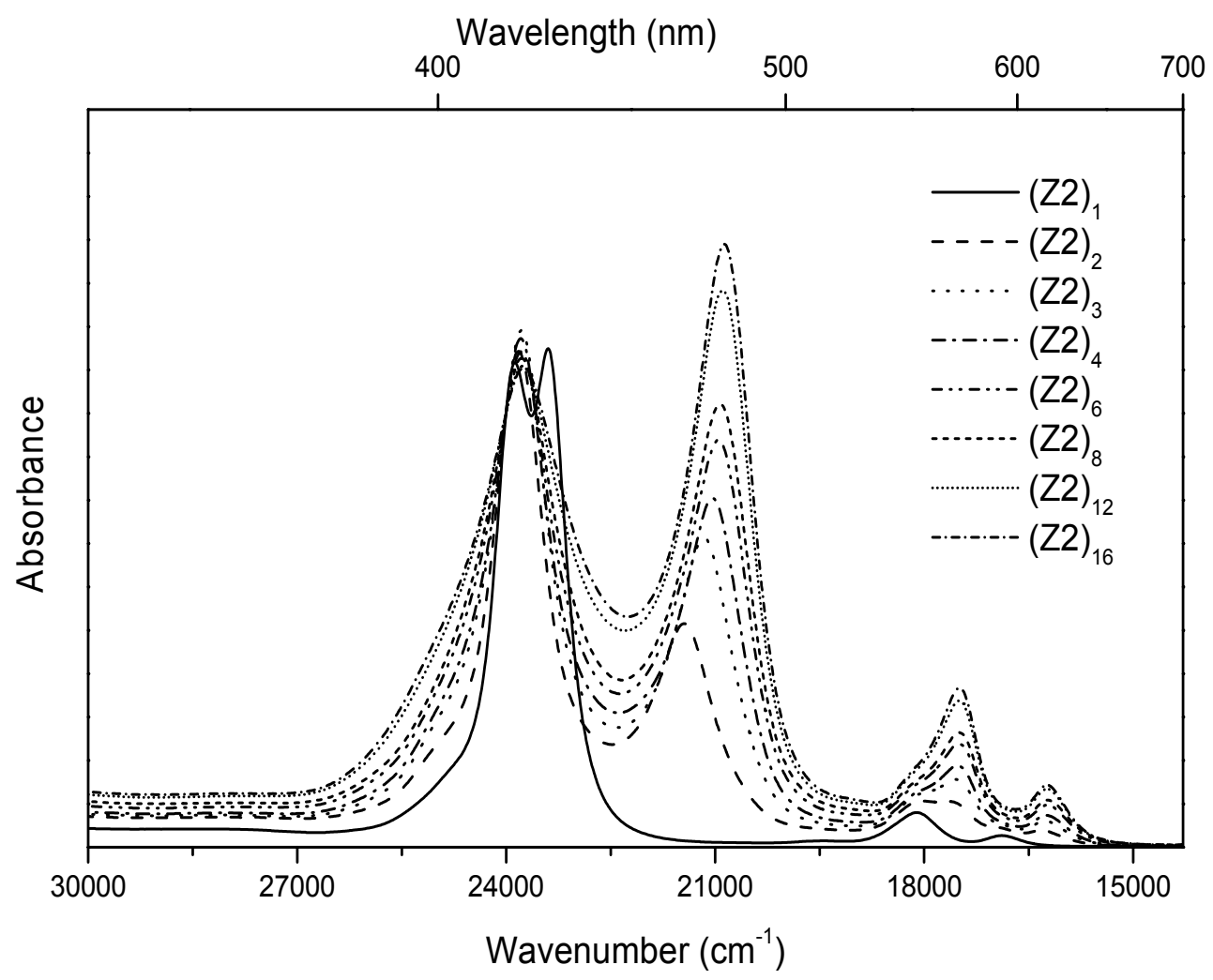

Figure S4. Absorption spectra of (Z2) $)_{\mathbf{n}}$ arrays in THF. 

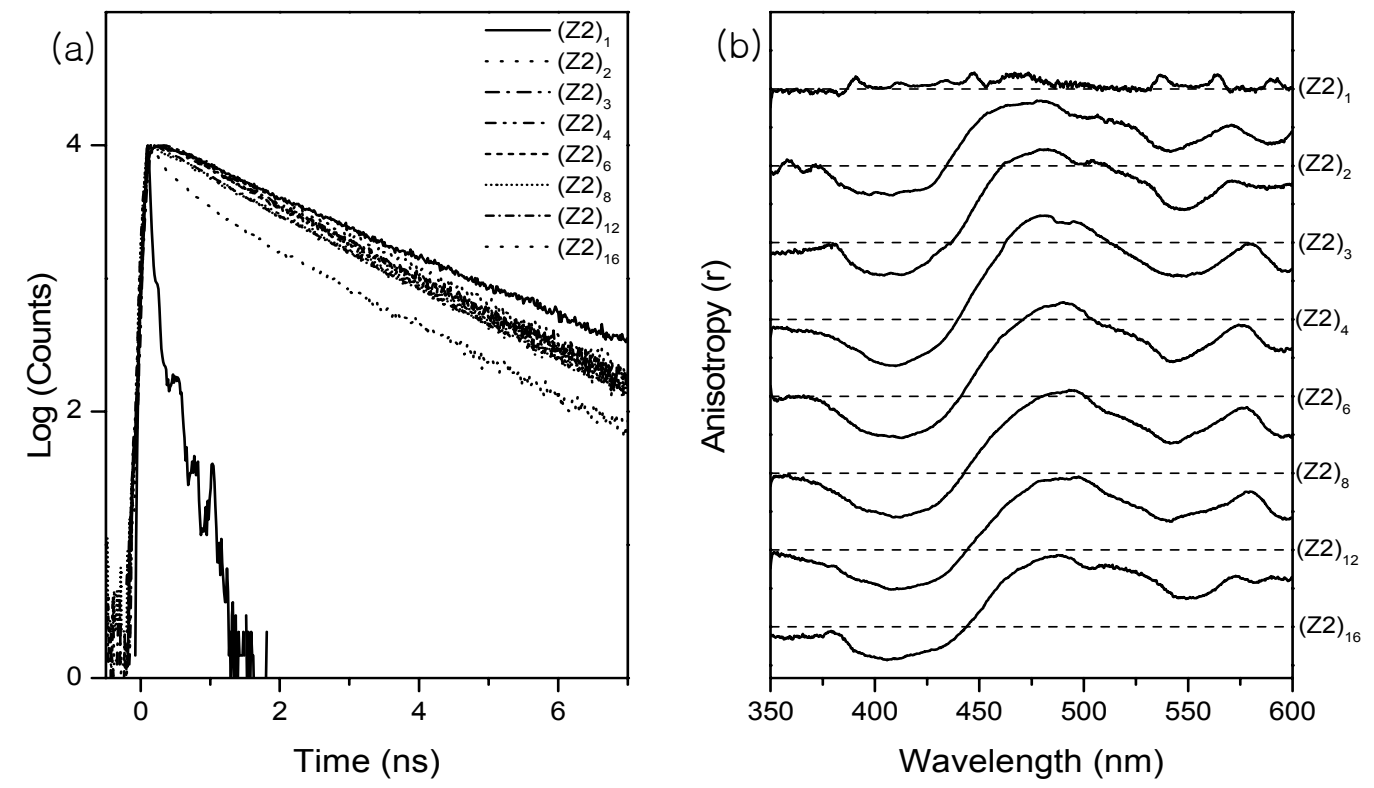

Figure S5. Fluorescence decay profiles (a) and fluorescence excitation anisotropy spectra (b) of (Z2) $)_{\mathbf{n}}$ in THF. 
Table S3. Soret Band Splitting Energies, Fluorescence Quantum Yields, Lifetimes, and Anisotropy Values of $(\mathbf{Z 2})_{\mathrm{n}}$

\begin{tabular}{llllllll}
\hline sample & $\Delta \mathrm{E}\left(\mathrm{cm}^{-1}\right)^{\mathrm{a}}$ & $\boldsymbol{\Phi}_{\mathrm{F}}{ }^{\mathrm{b}}$ & $\tau_{\mathrm{ave}}(\mathrm{ns})^{\mathrm{c}}$ & $\tau_{\mathrm{ave}}(\mathrm{ns})^{\mathrm{d}}$ & $\tau_{\mathrm{ave}}(\mathrm{ns})^{\mathrm{e}}$ & $\tau_{3}(\mathrm{~ns}, \%)^{\mathrm{f}}$ & $\boldsymbol{r}^{\mathrm{g}}$ \\
\hline$(\mathbf{Z 2})_{1}$ & 473 & 0.032 & 1.83 & $2.11(72.3)$ & $1.10(27.7)$ & 0.03 \\
$(\mathbf{Z 2})_{2}$ & 2380 & 0.076 & 1.66 & $1.77(59.5)$ & $1.51(40.5)$ & 0.13 \\
$(\mathbf{Z 2})_{3}$ & 2623 & 0.085 & 1.53 & $1.77(66.6)$ & $1.06(33.4)$ & 0.18 \\
$(\mathbf{Z 2})_{4}$ & 2753 & 0.094 & 1.56 & $1.73(76.5)$ & $0.99(23.5)$ & 0.20 \\
$(\mathbf{Z 2})_{6}$ & 2836 & 0.074 & 1.50 & $1.74(65.7)$ & $1.05(34.3)$ & 0.18 \\
$(\mathbf{Z 2})_{8}$ & 2862 & 0.062 & 1.42 & $1.67(78.9)$ & $0.48(21.1)$ & 0.16 \\
$(\mathbf{Z 2})_{12}$ & 2874 & 0.047 & 1.34 & $1.64(72.2)$ & $0.56(27.8)$ & 0.14 \\
$(\mathbf{Z 2})_{16}$ & 2885 & 0.029 & 0.68 & $1.63(31.0)$ & $0.50(22.0)$ & $0.14(47.0)$ & 0.14
\end{tabular}

${ }^{a}$ Soret band splitting energy. ${ }^{\mathrm{b}}$ Fluorescence quantum yields. ${ }^{\mathrm{c}}$ Fluorescence decay lifetimes averaged with amplitude ratios. ${ }^{\mathrm{d}}$ Longer fluorescence lifetimes with amplitude ratios. ${ }^{\text {eff }}$ Shorter fluorescence lifetimes with amplitude ratios. ${ }^{\mathrm{g}}$ Maximum values at fluorescence excitation anisotropy spectra. 


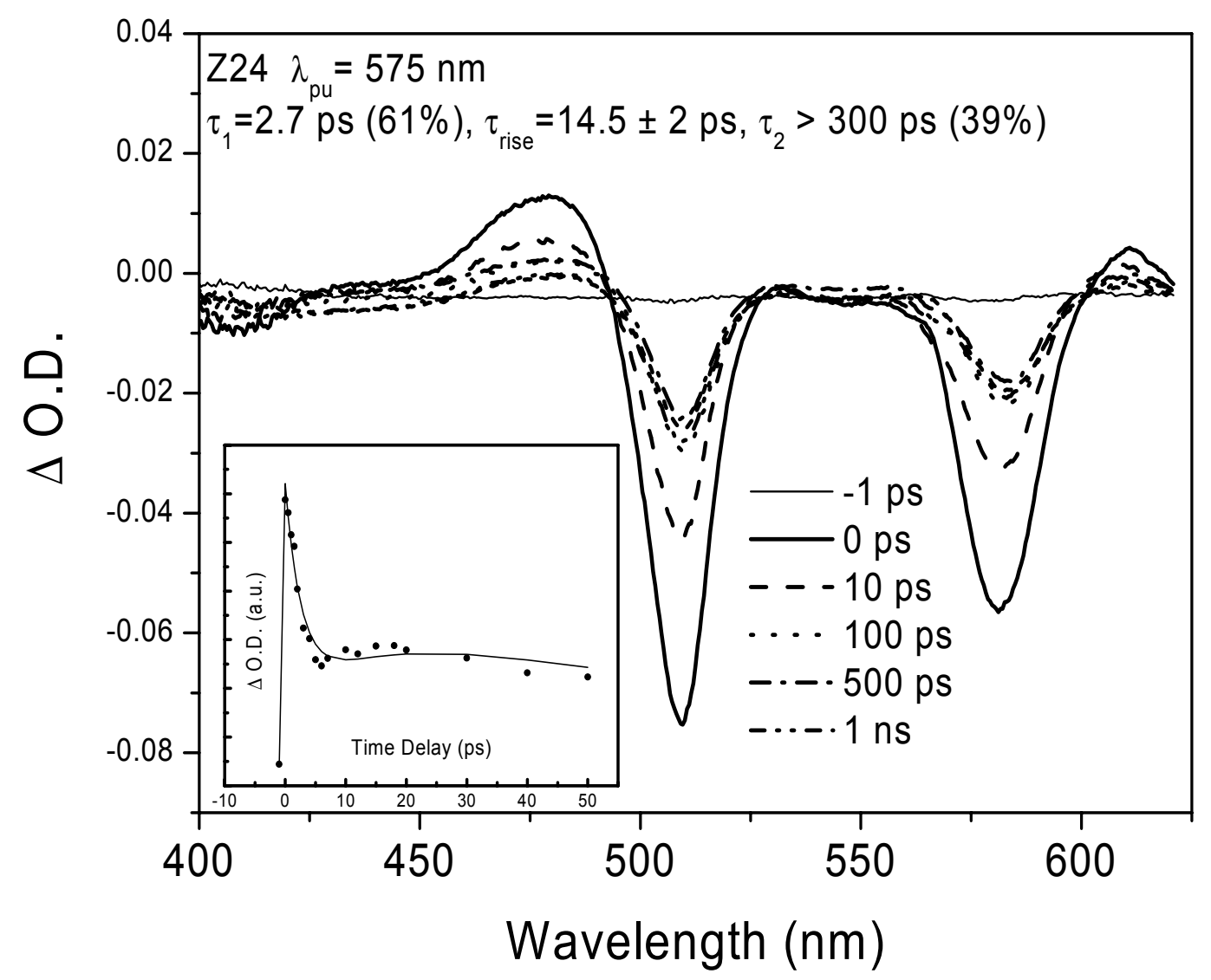

Figure S6. Transient absorption spectra of $\mathbf{Z 2 4}$ at various time delays in toluene. Inset shows the temporal profile at $588 \mathrm{~nm}$.

\section{Reference}

1. Kakitani, T.; Kimura, A., J. Phys. Chem. A 2002, 106, 2173.

2. Aratani, N; Cho, H. S.; Cho, S.; Ahn, T. K.; Kim, D.; Sumi, H.; Osuka, A., J. Am. Chem. Soc. 2003, 125, 9668. 